\title{
Vegetação endêmica e espécie invasora em campos rupestres de áreas garimpadas
}

\author{
Endemic vegetation and invasive species in rupestrian grasslands on mining sites
}

\author{
Abel Augusto Conceição ${ }^{1,2,3}$, Fabiciana da Hora de Cristo ${ }^{1}$, Alex de Almeida dos Santos ${ }^{1}$, Juliana Barbosa \\ dos Santos ${ }^{1}$, Emile Lemos Freitas ${ }^{1,2}$, Bárbara Paula dos Santos Borges ${ }^{1,2}$, Leonardo Silva Santa Rosa \\ Macêdo ${ }^{1}$ \& Regina Célia da Silva Oliveira ${ }^{1,2}$
}

\begin{abstract}
Resumo
Campos rupestres constituem uma vegetação típica de montanhas da Cadeia do Espinhaço e com elevado grau de endemismo de plantas, contendo algumas áreas com histórico de perturbações por garimpo e pisoteio. O presente estudo foca em duas perguntas principais: 1) Campos rupestres que foram perturbados pelo garimpo há cerca de 15 anos atrás possuem composição florística e estrutura similares a áreas sem perturbação? 2) A riqueza e abundância de espécies exóticas invasoras e de espécies nativas de ampla distribuição tendem a ser mais elevadas nessas áreas garimpadas ou pisoteadas? Quatro campos rupestres foram amostrados em Igatu, Andaraí, Chapada Diamantina, Bahia, Brasil: dois onde a atividade de garimpo cessou há 15 anos, um sob perturbação atual por pisoteio, mas sem histórico de garimpo e outro em uma área conservada (vegetação amostrada por 16 parcelas de 10x10 m, quatro em cada área). A distribuição geográfica das espécies foram determinadas com base na literatura e análises de classificação e ordenação foram feitas. A composição florística dos campos rupestres foi afetada pelas perturbações, mas apenas a perturbação por garimpo teve efeito marcante sobre a estrutura da vegetação. Espécies de ampla distribuição mais generalistas e a espécie invasora Melinis minutiflora P.Beauv. foram restritas às áreas perturbadas, mostrando a necessidade de monitoramento de espécies invasoras nas áreas garimpadas do Parque Nacional da Chapada Diamantina. Palavras-chave: Chapada Diamantina; conservação da biodiversidade; espécie ruderal; perturbação; regeneração vegetal
\end{abstract}

\begin{abstract}
The rupestrian grasslands vegetation occurs at the mountaintops of the Espinhaço Range harboring high number of endemic plant species. Despite its importance, some areas of rupestrian grasslands are under anthropogenic disturbance due to mining and trampling. The present study had two main questions: 1) Are the floristic composition and structure of rupestrian grasslands in areas impacted by mining similar to areas not subjected to mining? Are there tendencies to increase the abundances of exotic invasive and wild widespread plant species in areas impacted by mining or trampling? To evaluate our questions we sampled four rocky grasslands in Igatu, Andaraí, Chapada Diamantina, Bahia, Brazil: two areas where the mining activities stopped 15 years ago, one area under current disturbance due to trampling, but without mining historic, and a fourth area which is well conserved. The vegetation was sampled in 16 10x10 m plots, four in each site. The geographic distribution of each species was defined based in the literature. Cluster and ordination analysis were performed. We conclude that the floristic composition of the sites was affected by previous and current disturbances, but only the mining disturbance had strong effect in vegetation structure, with the conserved area harboring a great part of the Brazilian endemic species. The exotic and invasive plant Melinis minutiflora P.Beauv. occurred only at the disturbed sites, mainly in the mined sites, stressing that rupestrian grassland sites subjected to mining in the past should be monitored against invasive species.
\end{abstract}

Key words: Chapada Diamantina; biodiversity conservation; ruderal species; disturbance; vegetation regeneration.

\footnotetext{
Este artigo possui material suplementar disponível em http://dx.doi.org/10.6084/m9.figshare.1452960

${ }^{1}$ Universidade Estadual de Feira de Santana, Depto. Ciências Biológicas, Av. Transnordestina s/n, 44036-900, BA, Brasil.

${ }^{2}$ Universidade Estadual de Feira de Santana, Programa de Pós-graduação em Recursos Genéticos Vegetais da UEFS, Depto. Ciências Biológicas, Av.

Transnordestina s/n, 44036-900, BA, Brasil

${ }^{3}$ Autor para correspondência: abe118@gmail.com
} 


\section{Introdução}

Os campos rupestres são associados aos substratos de quartzito e hematita encontrados em montanhas, principalmente na Cadeia do Espinhaço em Minas Gerais e Bahia. Nestas áreas, ocorre um mosaico de comunidades vegetais, compostas predominantemente por espécies perenes herbáceas a arbustivas ocorrentes em solos arenosos, pedregosos ou sobre afloramentos rochosos. Os campos rupestres são especialmente importantes para conservação da biodiversidade, já que possuem elevada riqueza em espécies e alto grau de endemismos (Harley 1995; Giulietti et al. 1997; Conceição \& Giulietti 2002; Conceição \& Pirani 2005, 2007; Conceição et al. 2007a,b; Jacobi et al. 2007; Messias et al. 2011).

Apesar dos campos rupestres abrigarem número elevado de espécies endêmicas, eles estão e estiveram historicamente sujeitos a degradações derivadas da ação humana, como queimadas para renovação de pastagens, coleta de plantas com valor econômico e atividade garimpeira (Harley 1995; Giuletti et al. 1997; Jacobi et al. 2007; Santos et al. 2010). Usualmente, as perturbações favorecem o estabelecimento de plantas generalistas e/ou invasoras, com elevada habilidade competitiva, representando uma ameaça às espécies nativas endêmicas (Pivello et al. 1999; Meirelles et al. 1999; Lorenzi 2000; Porembski 2000; Hoffmann et al. 2004; Barbosa et al. 2010). Nos campos rupestres, a vulnerabilidade da vegetação nativa é acentuada, já que os solos formam bancos de sementes com baixo potencial regenerativo (Medina \& Fernandes 2007).

Em Igatu, na Chapada Diamantina, houve um período de intensa exploração de diamantes, o que ocasionou profundas modificações ambientais, como alterações de drenagens e a retirada da vegetação, inicialmente realizada manualmente e depois por meio de queimadas, como parte da atividade garimpeira instalada na região por volta de 1850 (Santos et al. 2010). Apesar dos impactos negativos causados pelo garimpo em Igatu (Santos et al. 2010), estudos na Chapada Diamantina demonstram que os campos rupestres são resilientes à perturbação decorrente deste tipo de atividade (Neves \& Conceição 2007) e ao distúrbio por incêndios (Neves \& Conceição 2010).

O presente estudo foi desenvolvido com base na hipótese de que a vegetação de campos rupestres estabelecida em áreas garimpadas no passado e em repouso há cerca de 15 anos deve apresentar estrutura similar à de áreas de campos rupestres não perturbadas pelo garimpo, porém possuindo diferenciação florística relacionada à maior proporção de espécies nativas de ampla distribuição e de espécies invasoras em detrimento de espécies endêmicas.

A partir dessa hipótese foram formuladas duas perguntas para serem respondidas no presente estudo: 1) Os campos rupestres perturbados pelo garimpo há 15 anos possuem composição e estrutura similares aos campos rupestres sem perturbação pelo garimpo? 2) O número de espécies e a abundância de espécies invasoras e de espécies nativas ruderais tendem a ser mais elevados nessas áreas garimpadas?

\section{Material e Métodos}

As áreas de estudo situam-se na vila de Igatu, município de Andaraí, Chapada Diamantina, Bahia, Brasil, localizada em uma importante área de exploração de diamantes no século XIX, cujo apogeu foi entre 1900 e 1920, quando possuía cerca de 30 mil habitantes. Entre 1996 e 1998, o garimpo na região foi banido e atualmente a vila de Igatu possui cerca de 400 habitantes e é tombada pelo Instituto do Patrimônio Histórico e Artístico Nacional, com o garimpo quase totalmente substituído pelo turismo (Nolasco 2000 apud Santos et al. 2010).

Igatu localiza-se no interior do Parque Nacional da Chapada Diamantina, nas coordenadas $12^{\circ} 48^{\prime} 27^{\prime \prime}$ 'S e $41^{\circ} 19^{\prime} 53^{\prime \prime} \mathrm{O}$, a $756 \mathrm{~m}$ de altitude, em uma região com serras de superfícies irregulares, refletindo antigos dobramentos, sendo encontradas áreas planas com afloramentos rochosos ou neossolos arenosos. O clima na região varia de úmido a subúmido, a depender da localização topográfica, com estação chuvosa geralmente de outubro a março e seca de abril a setembro. A precipitação média anual típica varia entre 1200 a $1400 \mathrm{~mm}$, com temperaturas extremas de $0^{\circ} \mathrm{C}$ entre junho a agosto e $30^{\circ} \mathrm{C}$ de novembro a janeiro (Nolasco 2000 apud Santos et al. 2010).

A amostragem da vegetação foi estratificada em três áreas perturbadas, sendo duas delas por possuírem histórico de garimpo: o garimpo

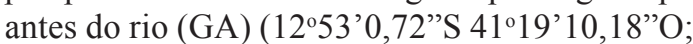
$730 \mathrm{~m}$ de altitude) e o garimpo depois do rio (GD) $\left(12^{\circ} 52^{\prime} 45,08^{\prime} \mathrm{S} 41^{\circ} 19^{\prime} 8,46^{\prime} \mathrm{O} ; 710 \mathrm{~m}\right.$ de altitude); e uma área perturbada não garimpada (PNG) (1253’40,63”S 4119'9,41”O; $740 \mathrm{~m}$ altitude), mas degradada por pisoteio e descarte 
de lixo, situada na vila e conhecida como Morro do Celular, frequentemente acessado pelos moradores e turistas para efetuar ligações de celular. Uma quarta área em local conservado (C) foi amostrada no local conhecido como Pedra do Descanso (1253'41,59"S-41'19'31,00"O; 900 m altitude). Nas duas áreas que foram garimpadas há 15 anos (GA e GD), a vegetação anterior à mineração é desconhecida, mas atualmente classificada como campo rupestre, onde há sinais de remoção de sedimento e rochas. Nas demais áreas predomina o hábitat de afloramento rochoso, assim como em outras áreas típicas de campos rupestres (Conceição \& Pirani 2005; Conceição et al. 2007a,b).

A vegetação foi amostrada por 16 parcelas 10x10 m em junho de 2012, quatro parcelas em cada área. Foi realizado o censo das espécies ocorrentes nas parcelas, constituindo os dados de composição florística (qualitativo). Em cada parcela de $10 \times 10 \mathrm{~m}$ foram sorteadas cinco subparcelas de $2 \times 2 \mathrm{~m}$, a partir das quais foram obtidos os dados de porcentagem de cobertura das espécies (Conceição \& Pirani 2005).

Os dados de composição de espécies levantados nas 16 parcelas de $10 \times 10 \mathrm{~m}$ foram incluídos na matriz de presença e ausência utilizada para análise da similaridade florística entre as parcelas utilizando o método de agrupamento Unweighted Pair Group Method with Arithmetic Mean (UPGMA) e coeficiente de Jaccard (Material suplementar 1). As porcentagens de cobertura levantadas nas cinco subparcelas de cada parcela de $10 \times 10 \mathrm{~m}$ foram somadas e arranjadas na matriz com os dados quantitativos por parcelas de $10 \times 10 \mathrm{~m}$ (Material suplementar 2), utilizados para análise de correspondência retificada (DCA) (Ter Braak 1995). Ambas as análises incluíram apenas as espécies ocorrentes em mais de uma parcela (Ter Braak 1995; McCune $\&$ Grace 2002) e foram processadas com uso do programa PAST 3.03, com produção dos gráficos cluster e biplot (DCA) (Hammer et al. 2001). A matriz incluindo todas as espécies também é disponibilizada (Material suplementar 3).

As espécies foram classificadas em famílias segundo o sistema do Angiosperm Phylogeny Group III (APG III 2009). A distribuição geográfica das espécies, assim como a caracterização quanto à ocorrência preferencial de algumas nativas em áreas antropizadas, foram definidas a partir da Lista de Espécies da Flora do Brasil. Espécies de plantas nativas ou exóticas que crescem preferencialmente em habitats submetidos a distúrbios humanos foram consideradas como ruderais e identificadas segundo Lorenzi (2000). O número de espécies ruderais ocorrentes nas subparcelas de $2 \times 2 \mathrm{~m}$, assim como suas porcentagens de cobertura, foram comparados entre as quatro áreas (GA, GD, PNG, C) por análise de variância (ANOVA), calculada com uso do programa PAST (Hammer et al. 2001).

\section{Resultados}

No total, foram amostradas 54 espécies nas 16 parcelas de $10 \times 10 \mathrm{~m}$, sendo $31 \mathrm{em} \mathrm{GA}$, 37 em GD, 30 em PNG e 25 em C (Tab. 1). Oito espécies ocorreram apenas no levantamento florístico feito nas parcelas de $10 \times 10 \mathrm{~m}$, mas não no levantamento fitossociológico feito nas subparcelas de $2 \times 2 \mathrm{~m}$. Destas oito espécies, quatro foram amostradas em $\mathrm{C}$, três em $\mathrm{PNG}$ e uma em GA e em GD (Tab. 1).

Ocorreram cinco espécies ruderais, nenhuma delas endêmica do Brasil. Essas cinco espécies foram restritas às áreas perturbadas, predominantemente nas duas áreas garimpadas ( $95 \%$ da cobertura de ruderais) (Tab. 2). Dessas, a exótica invasora Melinis minutiflora foi a espécie mais abundante, ocorrendo em todas as áreas perturbadas (GA, GD e PNG), mas não na área conservada.

Houve predomínio de espécies endêmicas do Brasil na riqueza total de todas as áreas, variando entre 17 a 22 espécies endêmicas por área (Tab. 3). As espécies endêmicas corresponderam a cerca de três quartos da porcentagem de cobertura de cada uma das áreas amostradas. Salienta-se a elevada abundância das espécies endêmicas da Bahia, principalmente em $\mathrm{C}$ e GA, onde essas endêmicas do estado representam mais da metade da cobertura vegetal.

Em C, ocorreu o menor número de espécies referidas para áreas antropizadas (2) e a maior cobertura delas $(14,9 \%)$, enquanto que nas áreas garimpadas ocorreu o maior número de espécies (6 a 8$)$ e a menor cobertura em GA (4,03\%) (Tabela 3). O número de espécies referidas para áreas antropizadas foi distinto apenas entre GD e $\mathrm{C}\left(F_{(3,12)}=3,638, p=0,0450\right)$, sendo menor em $\mathrm{C}(p=0,033)$. Não houve diferença significativa entre nenhuma das áreas quando consideradas as porcentagens de cobertura $\left(F_{(3,12)}=0,594, p\right.$ $=0,864)$. 


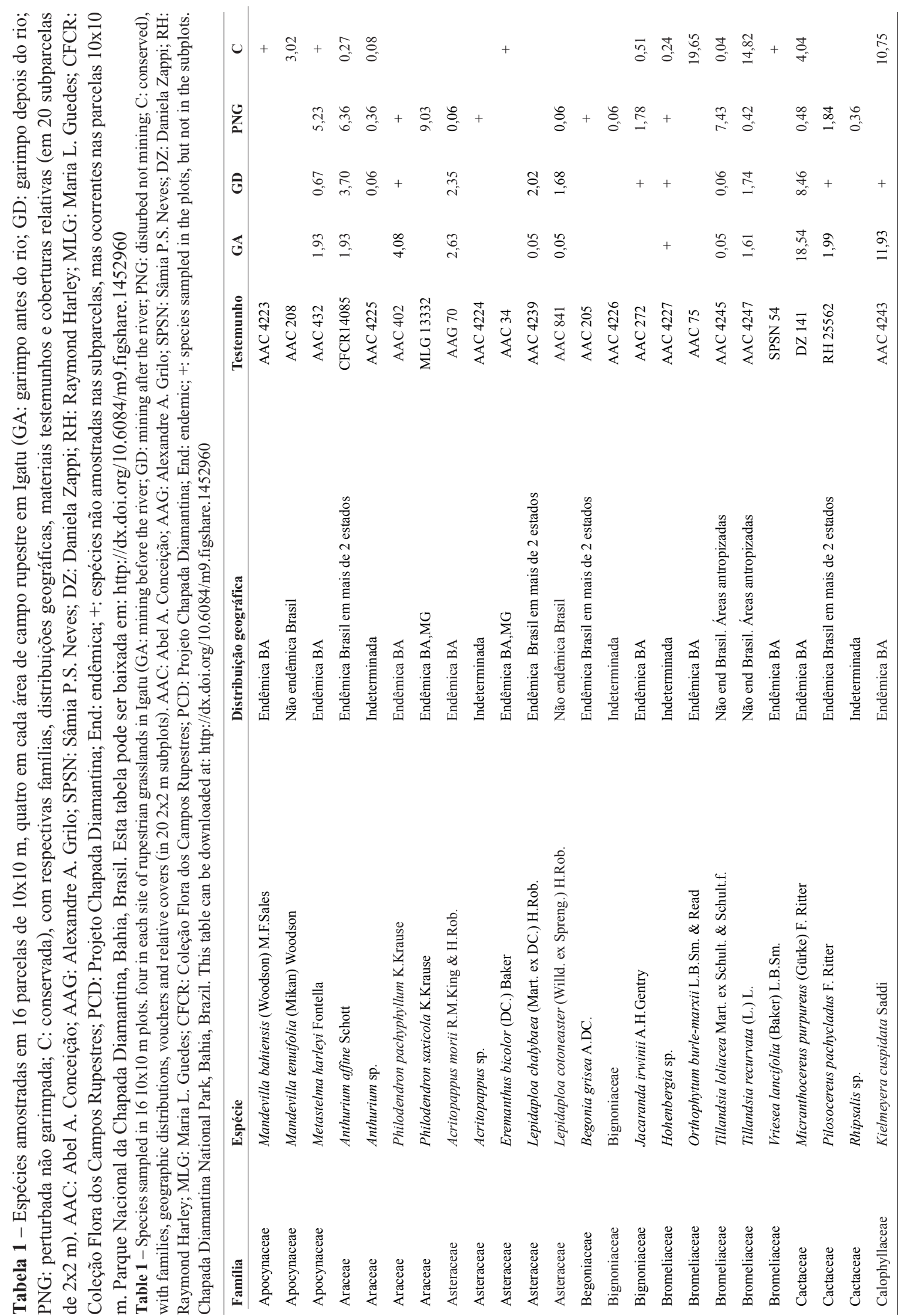




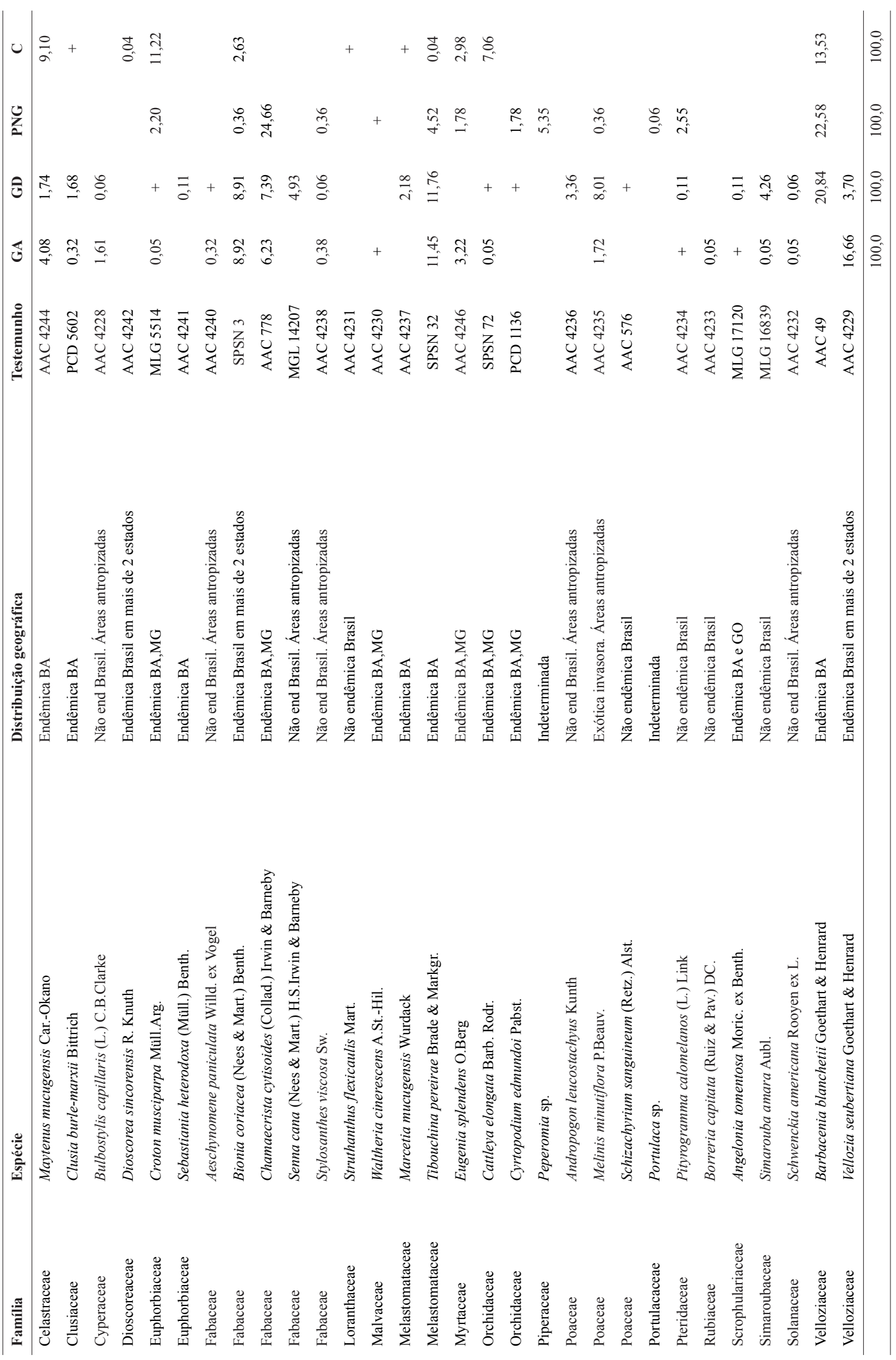


Tabela 2 - Cobertura relativa (\%) das espécies ruderais (Lorenzi 2000) amostradas em áreas perturbadas e não perturbadas (NP) em Igatu, Parque Nacional da Chapada Diamantina, Bahia, Brasil. GA: garimpo antes do rio; GD: garimpo depois do rio; PNG: perturbada não garimpada; $\mathrm{C}$ : conservada. * Refere-se à única espécie exótica invasora. Table 2 - Relative cover (\%) of ruderal species (Lorenzi 2000) sampled in disturbed and not disturbed (NP) areas in Igatu, Chapada Diamantina National Park, Bahia, Brazil. GA: mining before the river; GD: mining after the river; PNG: disturbed not mining; C: conserved. * Indicates the single invasive exotic species.

\begin{tabular}{lcccc}
\hline Espécies ruderais (Lorenzi 2000) & \multicolumn{3}{c}{ Perturbadas } & NP \\
\cline { 2 - 5 } & GA & GD & PNG & C \\
\hline *Melinis minutiflora P.Beauv. & 1,7 & 8,0 & 0,4 & 0,0 \\
Andropogon leucostachyus Kunth & 0,0 & 3,4 & 0,0 & 0,0 \\
Bulbostylis capillaris (L.) C.B.Clarke & 1,6 & 0,1 & 0,0 & 0,0 \\
Stylosanthes viscosa Sw. & 0,4 & 0,1 & 0,4 & 0,0 \\
Aeschynomene paniculata Willd. ex Vogel & 0,3 & 0,0 & 0,0 & 0,0 \\
Total & 4,0 & 11,6 & 0,8 & 0,0 \\
\hline
\end{tabular}

Tabela 3 - Número de espécies (Spp) e coberturas relativas (CR) por distribuição geográfica conforme a Lista de Espécies da Flora do Brasil. GA: garimpo antes do rio; GD: garimpo depois do rio; PNG: perturbada não garimpada; C: conservada. End.: endêmica. A.Antrop.: áreas antropizadas. Campos rupestres em Igatu, Parque Nacional da Chapada Diamantina, Bahia, Brasil.

Table 3 - Species number (Spp) and relative covers (CR) per geographic distribution according the Brazilian Flora Checklist. GA: mining before the river; GD: mining after the river; PNG: disturbed not mining; C: conserved. End.: endemic. A.Antrop.: anthropic areas. Rupestrian grasslands in Igatu, Chapada Diamantina National Park, Bahia, Brazil.

\begin{tabular}{|c|c|c|c|c|c|c|c|c|}
\hline \multirow{3}{*}{ Distribuição geográfica } & \multicolumn{6}{|c|}{$\begin{array}{c}\text { Áreas } \\
\text { Perturbadas }\end{array}$} & \multirow{2}{*}{\multicolumn{2}{|c|}{$\begin{array}{c}\begin{array}{c}\text { Área não } \\
\text { perturbada }\end{array} \\
\text { C }\end{array}$}} \\
\hline & \multicolumn{2}{|c|}{ GA } & \multicolumn{2}{|c|}{ GD } & \multicolumn{2}{|c|}{ PNG } & & \\
\hline & Spp & $\mathrm{CR}$ & Spp & $\mathrm{CR}$ & Spp & $\mathrm{CR}$ & Spp & $\mathrm{CR}$ \\
\hline Endêmica da Bahia & 8 & 54,97 & 12 & 49,80 & 7 & 34,64 & 12 & 57,61 \\
\hline Endêmica de dois estados & 6 & 9,56 & 5 & 7,51 & 6 & 39,45 & 4 & 21,26 \\
\hline Demais endêmicas Brasil & 5 & 29,55 & 5 & 18,32 & 4 & 8,56 & 3 & 2,94 \\
\hline Não end. Brasil e A.Antrop. & 6 & 4,03 & 8 & 10,25 & 4 & 8,2 & 2 & 14,86 \\
\hline Demais não end. Brasil & 4 & 1,72 & 4 & 6,05 & 1 & 2,61 & 2 & 3,02 \\
\hline Espécie exótica invasora & 1 & 1,72 & 1 & 8,01 & 1 & 0,36 & 0 & 0 \\
\hline Indeterminada & 2 & 0,00 & 1 & 0,06 & 7 & 6,18 & 2 & 0,31 \\
\hline Total & 31 & 100 & 37 & 100 & 30 & 100 & 25 & 100 \\
\hline
\end{tabular}

O dendrograma de similaridade de Jaccard (Fig. 1) revelou $21 \%$ de similaridade florística entre as 16 parcelas e discerniu as parcelas em área conservada (C) das parcelas em áreas perturbadas (GA, GD e PNG). O grupo das parcelas em C apresenta $37 \%$ de similaridade, enquanto o das parcelas em áreas perturbadas apresenta $24 \%$ de similaridade. As parcelas mais similares entre si foram em PNG, formando pares $56 \%$ e $66 \%$ similares.
As parcelas das áreas perturbadas ( $\mathrm{G}$ e PNG) não ficaram completamente separadas das parcelas da área conservada, como pode ser observado na Figura 2, embora haja tendência na formação de grupos. Com exceção de GD2, o eixo 1 separa as parcelas das áreas garimpadas das demais. As parcelas GD2 e PNG1 misturamse às parcelas da área C. As parcelas em PNG são distinguidas pelo eixo 2 da Figura 2. 


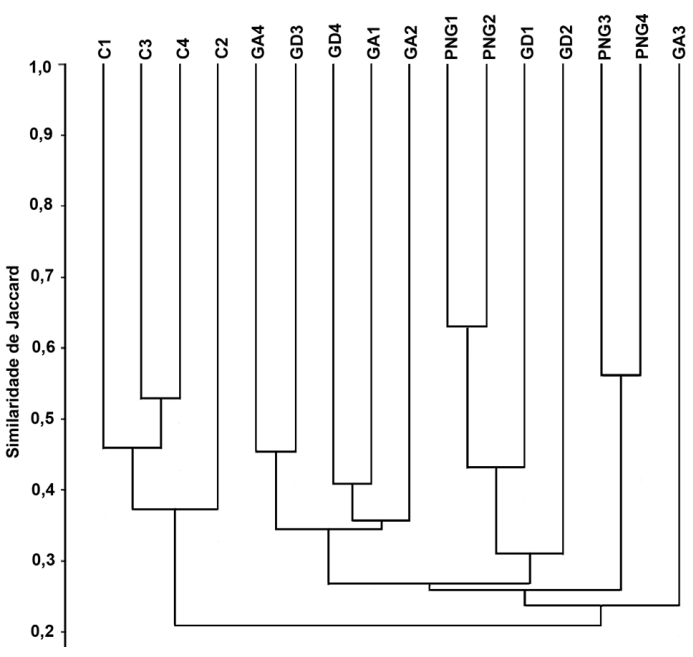

Figura 1 - Dendrograma de similaridade de Jaccard e UPGMA agrupando as 16 parcelas de $10 \times 10 \mathrm{~m}$ em campos rupestres de Igatu, Parque Nacional da Chapada Diamantina, Bahia, Brasil. Correlação cofenética $=0,7$. GA: garimpo antes do rio; GD: garimpo depois do rio; PNG: perturbada não garimpada; $\mathrm{C}$ : conservada.

Figure 1 - Cluster of $1610 \times 10$ m plots using Jaccard's similarities and UPGMA. Cophenetic correlation $=0.7$. GA: mining before the river; GD: mining after the river; PNG: disturbed not mining; C: conserved. Rupestrian grasslands in Igatu, Chapada Diamantina National Park, Bahia, Brazil.

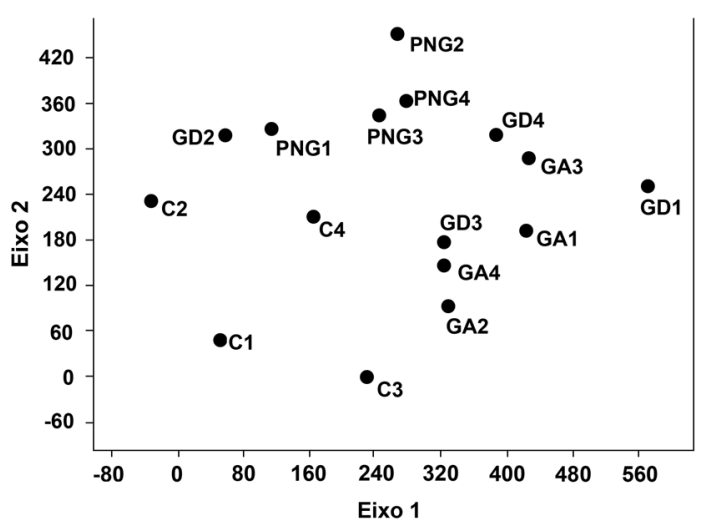

Figura 2 - Análise de correspondência retificada com base na matriz de áreas de coberturas das espécies nas 16 parcelas de $10 \times 10 \mathrm{~m}$ nos campos rupestres em Igatu, Parque Nacional da Chapada Diamantina, Bahia, Brasil. Autovalor do eixo $1=0,73$ e do eixo $2=0,45$. GA: garimpo antes do rio; GD: garimpo depois do rio; $\mathrm{PNG}$ : perturbada não garimpada; $\mathrm{C}$ : conservada.

Figure 2 - Dentrended Correspondence Analysis based in a matrix with species cover areas in $1610 \times 10 \mathrm{~m}$ plots in the rupestrian grasslands in Igatu, Chapada Diamantina National Park, Bahia, Brazil. Axis 1 eigenvalues $=0.73$ and axis $2=0.45$. GA: mining before the river; GD: mining after the river; PNG: disturbed not mining; $\mathrm{C}$ : conserved.

\section{Discussão}

A vegetação da área conservada possui uma flora diferenciada, tanto das áreas perturbadas pelo garimpo (GA e GD) como das áreas afetadas pelo pisoteio humano (PNG). Essa distinção deve ser consequência das perturbações, com eliminação de algumas espécies mais sensíveis e introdução de espécies ruderais e da exótica Melinis minutifolia, que foram exclusivas das áreas perturbadas.

Ao contrário do esperado, as espécies referidas para áreas antropizadas tenderam a apresentar coberturas relativas maiores nos campos rupestres da área conservada, embora representadas por apenas duas espécies de Bromeliaceae (Tillandsia recurvata e Tillandsia loliaceae). $\mathrm{Na}$ verdade, essa abundância elevada deve-se apenas a $T$. recurvata $(99,7 \%$ da cobertura de espécies referidas para áreas antropizadas na área conservada - 14,8\% da cobertura total desta área), que é um componente usual em campos rupestres sem perturbação (Conceição \& Pirani 2005; Jacobi \& Carmo 2011), não sendo ruderal por não ter ocorrência preferencial em habitats submetidos a distúrbios humanos.

Apesar das distinções reveladas pelo dendrograma, a análise de correspondência não revelou grupos exclusivos em $\mathrm{C}$, já que a parcela GD2 é mais similar às parcelas em C (Fig. 2). Isso revela que mesmo em áreas garimpadas há parte da vegetação estruturalmente similar à vegetação em áreas conservadas (Fig. 2), como é o caso da vegetação na parcela GD2, composta por $100 \%$ de espécies endêmicas. Por outro lado, as demais parcelas nas áreas garimpadas formam um grupo e são evidências de que a perturbação pelo garimpo afeta a estrutura da vegetação, já que ainda são distintas das parcelas sob perturbação, mas sem garimpo.

O comprimento dos eixos do DCA revela que há um forte gradiente entre as parcelas analisadas. $\mathrm{O}$ eixo 1 pode ser interpretado como um gradiente de perturbação pelo garimpo, sendo polarizado por GD1, uma parcela altamente impactada, que possui quase metade da cobertura vegetal composta pela exótica invasora $M$. minutiflora (45\%), que juntamente com outras duas espécies ruderais (Andropogon leucosthachyus e Bulbostyllis capillaris), compreendem $65 \%$ da vegetação nessa parcela. Na outra extremidade do eixo está a parcela C2, com 100\% da vegetação endêmica da Bahia e Minas Gerais, sendo 78\% delas endêmicas da Bahia. 
Melinis minutiflora (capim-gordura) foi uma espécie exótica invasora presente nas áreas impactadas e é uma ameaça à biodiversidade nas savanas e campos, principalmente nas áreas com elevada luminosidade (Pivello et al. 1999; Hoffmann et al. 2004), assim como nos afloramentos rochosos, aumentando o risco de incêndios no período seco (Meirelles et al. 1999; Porembski 2000; Hoffmann et al. 2004), motivo pelo qual é especialmente indesejável nas estradas de ferro (Lorenzi 2000). Apesar dessa espécie ser uma exótica invasora, ela não estava presente em diversas comunidades de campos rupestres estudadas (Conceição \& Giulietti 2002; Conceição \& Pirani 2005; Conceição et al. 2007; Jacobi et al. 2008; Vincent \& Meguro 2008; Ataíde et al. 2011; Borges et al. 2011; Messias et al. 2011), mas estava presente em áreas afetadas por garimpo, tanto em Igatu (presente estudo) como em outros campos rupestres da Bahia (Neves \& Conceição 2007) e Minas Gerais (Jacobi et al. 2007), assim como em áreas próximas a rodovias (Barbosa et al. 2010), ameaçando a biodiversidade desse ecossistema. Eventualmente, M. minutiflora ocorre em campos rupestres sem garimpo, como nas cangas da Serra da Calçada, em Minas Gerais, mas com abundância marcante apenas nas áreas antropizadas (Viana \& Lombardi 2007).

A ausência de M. minutiflora em áreas afetadas por incêndios (Neves \& Conceição 2010) é uma evidência do fogo como fator natural dos campos rupestres e salienta as diferenças dos campos rupestres estabelecidos em áreas garimpadas. Não é possível saber se essa espécie está em processo de expansão nos campos rupestres de Igatu, ameaçando essa vegetação na região, ou se $M$. minutiflora está sendo gradativamente excluída nessas áreas em regeneração há 15 anos da interrupção do garimpo. Ao considerar as alterações no substrato e redução da cobertura vegetal nas áreas garimpadas (Santos et al. 2010), o restabelecimento de uma vegetação endêmica em 15 anos salienta o elevado potencial de regeneração natural dos campos rupestres (autóctone).

Em síntese, conclui-se que a composição florística foi afetada pelas perturbações, sejam as decorrentes do garimpo, sejam as resultantes do pisoteio de turistas e moradores. No entanto, apenas a perturbação por garimpo teve efeito mensurável marcante sobre a estrutura da vegetação. A maior parte da vegetação é composta por espécies endêmicas do Brasil, sempre com elevada proporção de espécies restritas à Bahia.
As ausências de espécies ruderais e da exótica M. minutiflora nos campos rupestres em áreas conservadas, ao contrário do que ocorre nas áreas perturbadas, são evidências de que os impactos antrópicos aumentam a susceptibilidade da vegetação à ocorrência de espécies ruderais e invasoras. Desse modo, as áreas garimpadas no Parque Nacional da Chapada Diamantina merecem atenção especial para conservação da biodiversidade, justificando um monitoramento de espécies invasoras nessas áreas.

\section{Material suplementar}

Arquivos suplementares a este artigo estão disponíveis. S1 corresponde à matriz de presençaausência utilizada na análise de agrupamento da Figura 1, onde apenas espécies presentes em duas ou mais parcelas estão representadas e S3 corresponde à mesma matriz com todas as espécies coletadas (incluindo as espécies coletadas em apenas uma parcela). S2 corresponde à matriz com a cobertura de cada espécie amostrada nas parcelas fitossociológicas, utilizadas para criar o gráfico de ordenação da Figura 2. O último arquivo suplementar é a Tabela 1 deste artigo em formato de texto para download.

\section{Agradecimentos}

Os autores agradecem à Aline P. Nunes, Mariana M. Luz e Karolyne N. Soares, a contribuição no processamento dos dados; à dois revisores anônimos e ao editor Marcelo Moro, as sugestões; ao $\mathrm{CNPq}$, a bolsa de produtividade em pesquisa do primeiro autor (311301/2011-8) e ao SISBIO, a licença de coleta no Parque Nacional da Chapada Diamantina.

\section{Referências}

APG III. 2009. An update of the Angiosperm Phylogeny Group classification for the orders and families of flowering plants: APG III. Botanical Journal of the Linnean Society 161: 105-121.

Ataíde, E.S.; Castro, P.T.A. \& Fernandes, G.W. 2011. Florística e caracterização de uma área de campo ferruginoso no complexo minerário alegria, Serra de Antônio Pereira, Ouro Preto, Minas Gerais, Brasil. Revista Árvore 35: 1265-1275.

Barbosa, N.P.U.; Fernandes, W.F.; Carneiro, M.A.A. \& Júnior, L.A.C. 2010. Distribution of non-native invasive species and soil properties in proximity to paved roads and unpaved roads in a quartzitic mountainous grassland of southeastern Brazil (rupestrian fields). Biological Invasions 12: 3745-3755.

Borges, R.A.X.; Carneiro, M.A.A. \& Viana, P.L. 2011. Altitudinal distribution and species richness of 
herbaceous plants in campos rupestres the Southern Espinhaço Range, Minas Gerais, Brasil. Rodriguésia 62: 139-152.

Conceição, A.A. \& Giulietti, A.M. 2002. Composição florística e aspectos estruturais de campo rupestre em dois platôs do Morro do Pai Inácio, Chapada Diamantina, Bahia, Brasil. Hoehnea 29: 37-48.

Conceição, A.A. \& Pirani, J.R. 2005. Delimitação de habitats em campos rupestres na Chapada Diamantina: substratos, composição florística e aspectos estruturais. Boletim de Botânica da Universidade de São Paulo 23: 85-111.

Conceição, A.A. \& Pirani, J.R. 2007. Diversidade em quatro áreas de campos rupestres na Chapada Diamantina, Bahia, Brasil: espécies distintas, mas riquezas similares. Rodriguésia 58:193-206.

Conceição, A.A.; Giulietti, A.M. \& Meirelles, S.T. 2007a. Ilhas de vegetação em afloramentos de quartzito-arenito no Morro do Pai Inácio, Chapada Diamantina, Bahia, Brasil. Acta Botanica Brasilica 21: 335-347.

Conceição, A.A.; Pirani, J.R. \& Meirelles, S.T. 2007 b. Floristics, structure and soil of insular vegetation in four quartzite-sandstone outcrops of "Chapada Diamantina", Northeast Brazil. Revista Brasileira de Botânica 30: 641-656.

Giulietti,A.M.; Pirani, J.R. \& Harley, R.M. 1997. Espinhaço Range Region, Eastern Brazil. In: Davis, S.D.; Heywood, V.H.; Herrera-Macbryde, O.; Villa-Lobos, J. \& Hamilton, A.C. (eds.). Centres of plant diversity. A guide and strategy for their conservation. Vol. 3. The Americas. IUCN Publication Unit, Cambridge. Pp. 397-404.

Hammer, O; Harper, D.A.T. \& Ryan, P.D. 2001. Paleontological statistics software package for education and data analysis. Paleontologia Eletronica 4: 1-9.

Harley, R.M. 1995. Introduction. In: Stannard, B.L. (ed.). Flora of the Pico das Almas, Chapada Diamantina, Brazil. Royal Botanic Gardens, Kew. Pp. 1-42.

Hoffmann, W.A.; Lucatelli, V.M.P.C.; Silva, F.J.; Azeuedo, I.N.C.; Marinho, M.S.; Albuquerque, A.M.S.; Lopes, A.O. \& Moreira, S.P. 2004. Impact of the invasive alien grass Melinis minutiflora at the savana-forest ecotone in the Brazilian Cerrado. Diversity and Distributions 10: 99-103.

Jacobi, C.M.; Carmo, F.C.; Vincent, R.C. \& Stehmann, J.R. 2007. Plant communities on ironstone outcrops: a diverse and endangered Brazilian ecosystem. Biodiversity and Conservation 16: 2185-2200.

Jacobi, C.M.; Carmo, F.C. \& Vincent, R.C. 2008. Estudo fitossociológico de uma comunidade vegetal sobre canga como subsidio para a reabilitação de áreas mineradas no quadrilátero ferrífero, MG. Revista Árvore 32: 345-353.
Lista de Espécies da Flora do Brasil. Jardim Botânico do Rio de Janeiro. Disponível em $<$ http://floradobrasil. jbrj.gov.br/>. Acesso em 29 outubro 2014.

Lorenzi, H. 2000. Plantas daninhas do Brasil: terrestres, aquáticas, parasitas e tóxicas. $3^{\text {a }}$ ed. Instituto Plantarum, Nova Odessa. 608p.

McCune, B. \& Grace, J.B. 2002. Analysis of ecological communities. MjM, Oregon. 300p.

Medina, B.M.O. \& Fernandes, G.W. 2007. The potential of natural regeneration of rocky outcrop vegetation on rupestrian field soils in "Serra do Cipó", Brazil. Revista Brasileira de Botânica 30: 665-678.

Meirelles, S.T.; Pivello, V.R. \& Joly, C.A. 1999. The vegetation of granite rock outcrops in Rio de Janeiro, Brazil, and need for its protection. Environmental Conservation 26: 10-20.

Messias, M.C.T.B.; Leite, M.G.P.; Meira-Neto, J.A.A. \& Kozovits, A.R. 2011. Life-form spectra of quartzite and itabirite rocky outcrops sites, Minas Gerais, Brazil. Biota Neotropica 11: 255-268.

Neves, S.P.S. \& Conceição, A.A. 2007. Vegetação em afloramentos rochosos na Serra do Sincorá, Chapada Diamantina, Bahia, Brasil. Sitientibus Série Ciências Biológicas 7: 36-45.

Neves, S.P.S. \& Conceição, A.A. 2010. Campo rupestre recém-queimado na Chapada Diamantina, Bahia, Brasil: plantas de rebrota e sementes, com espécies endêmicas na rocha. Acta Botanica Brasilica 24: 697-707.

Porembski, S. 2000. The invasibility of tropical granite outcrops ('inselbergs') by exotic weeds. Journal of the Royal Society of Western Australia 83: 131-135.

Pivello, V.R.; Shida, C.N. \& Meirelles, S.T. 1999. Alien grasses in Brazilian savanas: a threat to the biodiversity. Biodiversity and Conservation 8: 1281-1294.

Santos, L.T.S.O.; Vasconcelos, M.P.; Rodrigues, D.P.; Nolasco, M.C. \& Jesus, T.B. 2010. Consequências da atividade garimpeira de diamante na Bacia do rio Coisa Boa, vila de Igatu, Andaraí, BA. Revista de Biologia e Ciências da Terra 10: 1-11.

Ter Braak, C.F.J. 1995. Ordination. In Jongman, R.H.G.; Ter Braak, C.J.F.; van Tongeren, O.F.R. (eds.). Data analysis in community and landscape ecology. Cambridge University Press, New York. Pp. 91-173.

Viana, P.L. \& Lombardi, J.A. 2007. Florística e caracterização dos campos rupestres sobre canga na Serra da Calçada, Minas Gerais, Brasil. Rodriguésia 58: 159-177.

Vincent, R.C. \& Meguro, M. 2008. Influence of soil proprieties on the abundance of plant species in ferruginous rocky soil vegetation, Southeastern Brazil. Revista Brasileira de Botânica 31: 377-388. 
